\title{
Clinical Concepts in Infantile Onset Epilepsy
}

\author{
Vivek Sirolia ${ }^{1} \cdot$ Prashant Jauhari $^{1}$ (B)
}

Received: 22 May 2019 / Accepted: 22 May 2019 / Published online: 3 June 2019

(C) Dr. K C Chaudhuri Foundation 2019

Infantile epilepsy is an intriguing disorder commonly associated with significant neurologic deterioration. In this issue of the Journal, the study by Sahu et al. has been published [1]. The authors must be appreciated for prospectively studying this group of disorders and coming up with a detailed description of seizure type, etiology, developmental status, neurological and other common medical comorbidities [1]. Their study shows that in an unselected group, a simple protocol comprising of clinical history, examination, neuroimaging and electroencephalography can provide an etiological yield comparable to the West. Perinatal asphyxia still remains the single most common etiology for infantile epilepsy as it is for cerebral palsy, global developmental delay or intellectual disability [2]. Other important facts brought out in this study are that a-third of cases were not diagnosed timely, $2 / 3 \mathrm{rd}$ had difficult to control seizures and majority (82\%) had developmental delay with severe to profound delay noticed in one-third of the study population. The study result urges that a more integrated and coordinated approach for quality care centered on the whole child is needed. The treatment plan should be overarching, simultaneously targeting seizure control, comorbid problems and neurological development.

Etiology and epilepsy syndrome are the two most common determinants of treatment outcome in infants with epilepsy. Epilepsy broadly results from a structural pathology (hypoxic insult, malformation), metabolic defect (vitamin responsive states, inborn errors) or a genetic variation (ion channelopathies) [3]. Epilepsy syndromes are unequivocally identifiable clustering of seizure semiology, electroencephalogram, age of presentation and outcome [4]. In agreement with the study finding that in the Indian set up majority of cases have a structural etiology, neuroimaging and electroencephalography should be the first step in the evaluation of infants with

Prashant Jauhari pjauhari0@gmail.com

1 Child Neurology Division, Department of Pediatrics, All India Institute of Medical Sciences, New Delhi 110029, India epilepsy. Once imaging is normal or non-specific or a strong family history is available, further investigations should be planned keeping the following points into consideration; not to miss a treatable etiology; identify the epilepsy syndrome, if any and early establishment of a genetic etiology. Therefore, a trial of vitamins namely pyridoxine, pyridoxal-5-phosphate, biotin and folinic acid should be given to all, especially when neuroimaging is inconclusive. The identified epilepsy syndrome such as West syndrome will further govern the treatment choices. Subsequent metabolic and genetic evaluation should be guided by the clinical course and preferably be ordered at centers equipped with facilities for managing such children. For conceptual understanding, encephalopathy predominant presentation often points towards a metabolic etiology while seizure predominant presentation with fever triggered seizures, hemiclonic seizures, seizures with changing laterality suggest an underlying ion channelopathy.

Another treatment guiding concept in epilepsy management is the presence or absence of an epileptic encephalopathy. In the study by Sahu et al., it is difficult to determine that whether the perinatal insult and/or subsequent structural damage led to all the identified clinical problems including seizures or the ongoing epilepsy itself propagated the seizures further and resulted in processes directly contributing to the observed developmental delay i.e., a state of epileptic encephalopathy. Epileptic encephalopathy is a self-sustaining state in which the ongoing epileptic activity perpetuates neurological damage leading to relentlessly occurring seizures and a variable degree of developmental delay/stagnation or regression beyond what is expected from the evident structural damage [5]. Management in such a case should be aggressive with pharmacological and/or non-pharmacological measures to break this vicious cycle of self-perpetuation.

The take home message should be that still greater awareness among physicians and parents is needed to decrease the delay in diagnosis of infantile onset epilepsies. To achieve an acceptable outcome, a structured algorithmic approach towards individual cases and organized holistic management services at equipped centers are required for this cohort of 
children. The evaluation protocol should be simple and focus upon the most prevalent etiologies. All attempts should be made not to miss treatable conditions.

\section{Compliance with Ethical Standards}

Conflict of Interest None.

\section{References}

1. Sahu PK, Mishra D, Juneja M, Taneja K. Clinico-etiological profile and developmental status of infants aged 1-24 mo with epilepsy. Indian J Pediatr. 2019. https://doi.org/10.1007/s12098-019-02943-2.
2. Jauhari P, Boggula R, Bhave A, et al. Aetiology of intellectual disability in paediatric outpatients in northern India. Dev Med Child Neurol. 2011;53:167-72.

3. Berg AT, Berkovic SF, Brodie MJ, et al. Revised terminology and concepts for organization of seizures and epilepsies: report of the ILAE commission on classification and terminology, 2005-2009. Epilepsia. 2010;51:676.

4. Commission on Classification and Terminology of the International League Against Epilepsy. Proposal for revised classification of epilepsies and epileptic syndromes. Epilepsia. 1989;30:389-99.

5. Jain P, Sharma S, Tripathi M, et al. Diagnosis and management of epileptic encephalopathies in children. Epilepsy Res Treat. 2013;2013:501981.

Publisher's Note Springer Nature remains neutral with regard to jurisdictional claims in published maps and institutional affiliations. 